\title{
Multilinguisme, plurilinguisme : un petit restaurant à Strasbourg, S'Zwellings Stuebel
}

Andrée Tabouret-Keller

\section{(2) OpenEdition}

\section{Journals}

Édition électronique

URL : http://journals.openedition.org/esp/421

DOI : 10.4000/esp.421

ISSN : 2532-0319

Éditeur

Centre d'Information sur l'Éducation Bilingue et Plurilingue

\section{Édition imprimée}

Date de publication : 1 décembre 2014

Pagination : 7-9

ISSN : 1127-266X

\section{Référence électronique}

Andrée Tabouret-Keller, « Multilinguisme, plurilinguisme : un petit restaurant à Strasbourg, S'Zwellings

Stuebel », Éducation et sociétés plurilingues [En ligne], 37 | 2014, mis en ligne le 01 octobre 2015,

consulté le 14 septembre 2020. URL : http://journals.openedition.org/esp/421 


\section{MULTILINGUISME, PLURILINGUISME: UN PETIT RESTAURANT À STRASBOURG, SZWELLINGS STUEBEL}

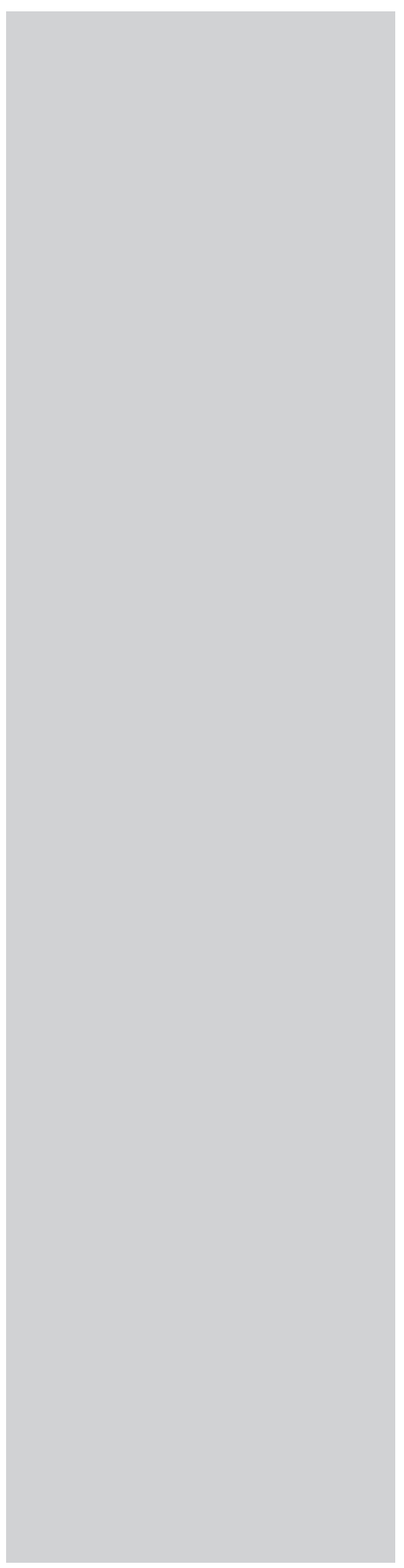

\section{Andrée TABOURET-KeLLER}

C e petit restaurant est non loin de la Gare SNCF dans une des rues qui partent en éventail de la place semi-circulaire de la gare vers le centre ville. Comme les autres, elle longe des restaurants, des hôtels, des agences de voyage.

La plupart des restaurants du quartier proposent des spécialités turques, grecques, pakistanaises, indiennes, libanaises, ou même chinoises ou japonaises. Le nôtre porte un nom en alsacien, $S^{\prime}$ Zwellings Stuebel, «la petite chambre des jumeaux». Le terme Stuebel n'a pas d'équivalent strict en français: en allemand le terme Stub ou Stubbe signifie "chambre», dans mon usage alsacien, le mot Stub signifie chambre à demeurer, celle où se trouve le Kachelofa, le poêle en briques vernissées, assez haut avec des compartiments où l'on peut faire mijoter un mets ou bien le garder au chaud. Dans les grandes fermes, ce poêle peut avoir deux parties en continue dont l'une est dans la cuisine adjacente et sert de cuisinière ordinaire. Le terme Stub, ou bien l'un de ses diminutifs, sont courants sur tout le nord de l'arc alpin, en bordure de la Suisse, de l'Allemagne, de l'Autriche. Mais d'où vient l'appellation de «jumeaux»? C'est que les propriétaires précédents étaient parents de deux petites filles jumelles, nées pendant qu'ils tenaient le restaurant (1).

Le restaurant a une petite salle au niveau de la rue et une autre à l'étage: le nombre de clients peut aller jusqu'à une cinquantaine; le restaurant est ouvert à midi en semaine et fermé le samedi et le dimanche. La clientèle est régulière, variable selon le jour de la semaine, plus abondante le mardi et le jeudi, elle provient essentiellement des nombreuse agences du quartier de la gare - de voyage, de location - mais aussi de l'un ou l'autre établissement institutionnel du quartier, la Bibliothèque municipale ou bien l'École d'architecture, ainsi que des agences de la SNCF elle-même, situées tout près de la gare. Nous y déjeunons, mon mari et moi, assez régulièrement. On y déjeune en moyenne pour un prix autour de 15 euros.

En l'espace d'un an, nous avons entendu le plus fréquemment le français, mais également l'alsacien, très peu souvent l'allemand, nous 
Multilinguisme, plurilinguisme: un petit restaurant à Strasbourg, S'Zwellings Stuebel

A. TABOURET-KeLLER avons vu une tablée s'entretenir en langue des signes, moins souvent nous avons entendu l'une ou l'autre langue slave, et peu de fois du roumain et du japonais, les commandes étant toujours faites en français. Avant les évènements du 11 septembre, des Américains venus avec leur arbre généalogique à la recherche de leurs ancêtres, étaient des clients réguliers, après cette date, leur présence s'est amoindrie. Il s'agit donc d'un établissement multilingue. Il arrive que le français et l'alsacien soient employés de concert à la même table mais pas de façon mélangée (pas de code-switching), plutôt de façon séparée pour une interpellation, ou bien entre deux voisins qui ont quelque chose de particulier à se dire, ou bien pour énoncer un Witz (pointe d'humour) que tout le monde comprend, que l'on va essayer de traduire pour celui «de l'intérieur» (1) tout en l'assurant qu'il ne peut sans doute pas vraiment l'apprécier.

Le restaurant est tenu par Monsieur Ma. B., né en 1962 en Lorraine du nord, et son épouse Madame Jo.Y., née en 1961 à Shanghaï. Le patronyme du patron est de consonance germanique comme la grande majorité des patronymes des régions de l'est, celui de son épouse est chinois. Le couple s'est connu en Alsace où a eu lieu le mariage dans un village local en 1991. M. Ma., cuisinier, avait entrepris avec un camarade un long voyage qui l'a mené jusqu'en Chine où il a séjourné six mois en 1985-86. Au retour de Chine, M. Ma. a entrepris des études à l'Alliance française avant de retourner à la cuisine. Le couple tient $S$ 'Zwellings Stuebel depuis 1998. Le restaurant dispose d'une aide pour la vaisselle avec qui la communication se fait en français.

M. Ma. n'est pas dialectophone, sa langue maternelle est le français, actuellement il a des notions de chinois, il «se débrouille pas mal» en anglais, langue qu'il a employée pendant son voyage en Asie. Il lit régulièrement la presse locale et nationale en français, achète parfois un journal anglais, il lit des revues «techniques» (son expression) de culture générale, de politique et de cuisine. Il aime lire, apprécie Camus, Duras, Verdier, ou H. Böll, à la télévision, il regarde les chaînes françaises. Mme Jo. a comme première langue le chinois de Shanghaï (c'est elle qui donne cette précision), sa scolarité s'est déroulée à Shanghaï, elle parle aujourd'hui couramment le français, non sans un léger accent. Elle parcourt la presse locale, elle ne lit guère de livres mais a une bonne connaissance d'un pan de la littérature française du 19ème siècle, connaît Maupassant, Zola, Alphonse Daudet qui ont été enseignés au lycée qu'elle fréquentait à Shanghaï «pour critiquer le régime capitaliste», précise-t-elle. Elle ne lit pas de revues, regarde ARTE, et des quantités de films en chinois, tant chinois que de toutes origines, car il y a en France un site Internet pour 
Multilinguisme, plurilinguisme: un petit restaurant à Strasbourg, S'Zwellings Stuebel

A. TABOURET-Keller

\section{NOTE}

(1) en alsacien de même qu'en allemand, Zweelling ou Zwilling ne permet pas de distinguer filles et garçons, il n'y a pas d'équivalent pour «jumeaux» et «jumelles». les Chinois. Leurs deux enfants sont nés en France, le garçon en 1993, la fille en 1994. Le garçon a appris à parler en chinois qu'il va perdre très vite, la fille en français. En 1996, Mme Jo. retourne pour six mois en Chine avec les deux enfants, à leur retour ils parlent couramment chinois mais aujourd'hui ils n'en ont plus que des notions. Ils font tous les deux des études, la fille prend des cours de chinois, elle a fait au lycée de l'allemand et de l'anglais, le garçon de l'allemand, ils donnent à l'occasion des coups de main pour le service au restaurant. La conversation familiale courante se tient en français, sauf, dit la maman, pour des «petits mots d'amour» ou pour des «engueulades». M. Ma. et les siens fréquentent sa famille, où les échanges se font en français, Mme Jo. rencontre de temps à autre un de ses cousins chinois lointains qui est en France dans le cadre d'un échange avec l'université de Hong Kong. Mme Jo. précise qu'en France elle ne se sent «pas vraiment» française, mais qu'en Chine elle ne se sent «plus vraiment» chinoise non plus. Il s'agit donc d'un groupe familial plurilingue, composé de personnes bilingues ou plurilingues.

Ainsi, multilinguisme de la situation particulière d'un restaurant dans ce quartier de la gare, et plurilinguisme des membres d'une famille d'origines et de pratiques linguistiques différentes, forment un agglomérat linguistique original qui se distingue par la bonne entente. 